\title{
Cache invalidation strategies for internet-based mobile ad hoc networks
}

\author{
Sunho Lim ${ }^{\mathrm{a}, *}$, Wang-Chien Lee ${ }^{\mathrm{b}}$, Guohong Cao ${ }^{\mathrm{b}}$, Chita R. Das ${ }^{\mathrm{b}}$ \\ a Department of Electrical Engineering and Computer Science, 210 Harding Hall, South Dakota State University, Brookings, SD 57007, USA \\ ${ }^{\mathrm{b}}$ Department of Computer Science and Engineering, The Pennsylvania State University, University Park, PA 16802, USA
}

Received 5 September 2006; received in revised form 16 February 2007; accepted 20 February 2007

Available online 2 March 2007

\begin{abstract}
Internet-based mobile ad hoc network (IMANET) is an emerging technique that combines a mobile ad hoc network (MANET) and the Internet to provide universal information accessibility. Although caching frequently accessed data items in mobile terminals (MTs) improves the communication performance in an IMANET, it brings a critical design issue when data updates. In this paper, we analyze several push and pull-based cache invalidation strategies for IMANETS. A global positioning system (GPS) based connectivity estimation (GPSCE) scheme is first proposed to assess the connectivity of an MT for supporting cache invalidation mechanisms. Then, we propose a pull-based approach, called aggregate cache based on demand (ACOD) scheme that uses an efficient search algorithm for finding the queried data items. In addition, we modify two push-based cache invalidation strategies, proposed for cellular networks, to work in IMANETS. They are called modified timestamp (MTS) scheme and MTS with updated invalidation report (MTS + UIR) scheme, respectively. We compare the performance of all these schemes as a function of query interval, cache update interval, and cache size through extensive simulation. Simulation results indicate that the ACOD scheme provides high throughput, low query latency, and low communication overhead, and thus, is a viable approach for implementation in IMANETS.
\end{abstract}

(c) 2007 Elsevier B.V. All rights reserved.

Keywords: Aggregate cache; Cache invalidation strategy; Internet-based mobile ad hoc networks; Global positioning system (GPS); Search algorithm

\section{Introduction}

Recent advances in high-speed wireless networks and Internet technologies have enabled wireless Internet networks, where users can access Internet services anywhere and anytime. Due to users' growing interests and falling cost in accessing the Internet wirelessly, Internet-based Mobile Ad hoc Networks, called IMANETs [8], has emerged to provide a ubiquitous communication infrastructure. An IMANET combines the wired Internet and wireless Mobile Ad hoc Networks (MANETS). In an IMANET, a mobile terminal $^{1}$ (MT) can connect to the Internet directly through

\footnotetext{
${ }^{*}$ Corresponding author. Tel.: +1 605688 5279; fax: +1 6056885880 . E-mail addresses: sunho.lim@sdstate.edu (S. Lim),wlee@cse.psu.edu (W.-C. Lee), gcao@cse.psu.edu (G. Cao),das@cse.psu.edu (C.R. Das).

${ }^{1}$ In this paper, we use the term mobile terminal (MT) to refer to a portable device (e.g. a laptop computer, a personal digital assistance (PDA), a mobile phone, a handheld computer, etc) or a person who carries it.
}

selected access points (APs) or indirectly through message relay via other MTs in wireless MANETS (e.g. IEEE 802.11 in ad hoc mode).

An IMANET is well suited for many Internet applications for its flexible accessibility and information availability. For example, in museums or shopping malls, users may need to access various types of information provided by local service providers through an electronic guide such as an info-station [37]. Users can dynamically form an ad hoc network to share information accessed from the infostation or from some selected access points (APs) to the Internet. However, frequent link disconnections and changes of network topologies (due to mobility of users) may reduce the information accessibility and increase the communication latency.

Caching frequently accessed data items in MTs is shown to be an effective techniques to enhance the communication performance in IMANETS. In a previous work [23], the authors proposed an aggregate cache for IMANETS where, 
by storing the data items in the local caches of the MTs and making them available to other MTs, members of the IMANET can efficiently access the data items. Thus, the aggregated local caches of the MTs can be considered as a large, unified cache for the IMANET. By using data items cached in the aggregate cache, we can reduce the average access delay, and thus, save the scarce wireless bandwidth. However, a critical design issue, cache invalidation, needs to be addressed for applications requiring strong data consistency with the server. When a data item in a server is updated, it is necessary to make sure that the cached copies of this data item are validated before they can be used.

Cache invalidation problem has been well studied in mobile computing environments [3,5,12,15,17,19,30,32, $35,38]$. However, these studies are all limited to cellular wireless networks in which the MTs only communicate with the base stations (BSs) but not with each other. In the cellular environment, a message from the BS can be delivered to all the MTs in one broadcast. This brings a great advantage to the server initiated invalidation methods. Indeed, many invalidation report (IR) based schemes were proposed in the literature to explore this advantage $[3,5,12,15,35,38]$. In these schemes, the server periodically broadcasts an IR. By knowing the arrival time of the IRs, the MTs may save energy by staying in the sleep mode or turning off their radio components.

Our study of cache invalidation in the IMANET environment is motivated by the following observations:

- The communication model in IMANET is different from that in the cellular environment - in IMANET, a multihop (instead of one-hop) communication is employed.

- The cost for a broadcast in IMANET is different from that in the cellular environment - a broadcast may lead to flooding of the whole network.

- The connectivity to server is less stable than that in the cellular environment - due to the user mobility, an MT may be disconnected or isolated from the server or other MTs.

Thus, the cache invalidation problem in an IMANET is unique from that in a cellular network due to multi-hop message relay, operation cost model, and uncertainty in message delivery.

In this paper, we examine several push and pull-based cache invalidation schemes for IMANETS. Our contributions are three-fold:

- First, in order to adapt and enhance these schemes for IMANET, we propose a method, called global positioning system-based connectivity estimation (GPSCE), for assessing the connectivity of an MT to the server (i.e., AP). With this enhancement, an MT can check whether it can access a server directly or indirectly through multihop relay.

- Second, we extend the search protocol used in [23] to better meet the requirements of cache invalidation schemes.
- Finally, with GPSCE and the modified search protocol, we adapt three existing cache invalidation schemes for IMANET: the first one, called aggregate cache-based on demand ( $A C O D$ ), is based on the pull strategy; while the other two, namely modified timestamp (MTS) and $M T S$ with updated invalidation report (MTS + UIR), are variants based on the push strategy.

We conduct simulation-based performance evaluation using $n s-2$ to observe the impact of query interval, cache update interval, aggregate cache size, and search algorithm on system performance. The results indicate that the pullbased ACOD strategy provides high throughput (successful query), low query latency, and low communication overhead. The revised search protocol, applicable to all cache invalidation schemes, helps in reducing the number of communication messages significantly. The overall results show that the ACOD cache invalidation scheme is superior in all aspects, and thus is a viable approach for implementation in IMANETS.

The rest of paper is organized as follows. The related work is reviewed in Section 2. The system model is introduced in Section 3, and the GPS-based connectivity estimation scheme is described in Section 4. The search algorithm and four cache invalidation schemes are presented in Section 5. Section 6 is devoted to performance evaluation, and the conclusions and future work are drawn in the last section.

\section{Related work}

There have been a lot of research efforts [3,5,12, $15,17,19,30,32,35,38]$ on the caching issue in wired and wireless networks. All caching schemes assume a stateful or a stateless server depending on whether it maintains the information about which data items are cached by which clients. In the stateful approach, when a data item is updated, the server sends an invalidation message to those clients who have the cached data item, e.g. Andrew File System (AFS) [19]. On the other hand, in the stateless approach, a client sends a message to the server to verify the validity of the cached data before the data item is used, e.g. Network File System (NFS) [32]. With this approach, it may incur high message traffic.

For peer-to-peer (P2P), Roussopoulos and Baker [30] suggested an update propagation mechanism. An intermediate client caches the index entries to locate the clients, where the contents are cached or stored, to reduce access latency and to balance the workload. By propagating the updated index entries, intermediate clients' index entries are maintained. However, cached index entries may become obsolete due to mobility of MTs that incurs changes of network topology in mobile environments.

Various cache invalidation schemes [3,5,12,15,17,35,38] have been suggested for cellular networks, where the MTs are one hop away from the BS or a server. Barbara and Inielinksi [3] proposed a scheme for a stateless server, 
where the server periodically broadcasts the invalidation report (IR), in which the updated data items are marked. Since the IR packet is broadcasted periodically, the client can operate in a doze mode and can wake up during the server broadcast to reduce the battery power. Depending on the report size, there is a tradeoff between the efficiency of cache invalidation and latency for a given communication bandwidth. Since the client should wait the IR packet before it answers a query, updated invalidation report (UIR) [5] is proposed to reduced the latency by adding updated items between IR packets. Also, in [38], broadcast channel error and downlink traffic issues are further considered. The timestamp (TS) scheme limits the report size by broadcasting only the updated data items during a window of $w$ IR intervals ( $w$ being a fixed parameter). Most of the prior approaches $[5,12,15,35,38]$ are variations of the TS scheme. In [17], an asynchronous scheme (AS) is suggested, where the server broadcasts an IR whenever a data item is updated and saves it at the home location cache (HLC), located in the server. However, none of these techniques can be directly applied to an IMANET, where an MT may multi-hops away from the server.

For MANETS, Hara [11] suggested a replication scheme for periodically updated data items, based on the previously suggested replica allocation method [10]. Replicated data items are reallocated periodically depending on the access frequency, remaining time to next update, and network topology. However, estimation of optimal reallocation period and remaining time to the next update are not practically feasible. In [18], a data dissemination strategy has been suggested in the presence of network partitions in MANETS, where a subset of MTs (quorum) is selected as a server. Since not all the servers in the quorum are reachable from the MTs, several heuristics are suggested to make the update/query operations efficient. For the similar purpose, a probabilistic quorum system for ad hoc networks [25] has been proposed to provide a reliable storage for query and update operations. Also, in [40], modified IR-based cache invalidation scheme with a cooperative cache [39] is proposed for cache consistency against a possible security attack in a MANET.

For the IMANET environment, Sailhan and Issarny [31] proposed a cooperative caching scheme to increase data accessibility by peer-to-peer communication among the MTs when they are out of bound of a fixed infrastructure. Papadopouli and Schulzrinne [26] proposed the 7DS architecture, in which several protocols are defined to share and disseminate information among users. It operates either in a prefetch mode, based on the information popularity and user's future needs or in an ondemand mode, which searches for data items in one hop multicast basis. Depending on the collaborative behavior, a peer-to-peer and server-to-client model are used. Unlike our propose, they mainly focus on data dissemination, and thus, cache invalidation and cache update issue are not explored.

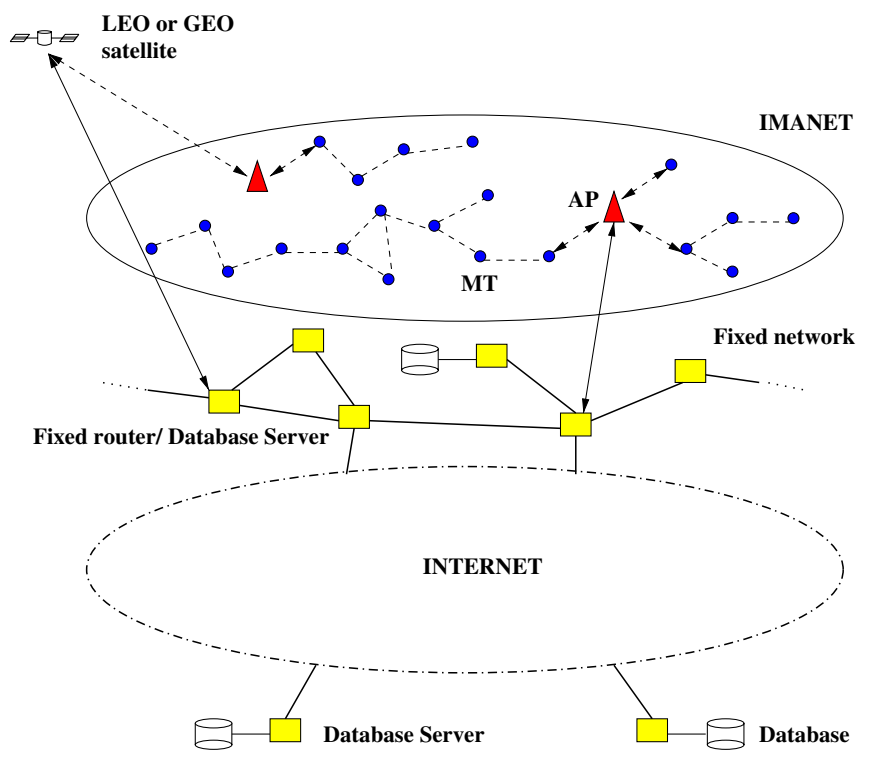

Fig. 1. A system model of Imanet.

\section{System model}

In this section, we describe the system model of IMANET, which is aimed at increasing both connectivity and accessibility of MTs to the wired Internet. We assume that an MT can not only connect to the Internet but also can forward a message for communication with other MTs via a wireless LAN (e.g. IEEE 802.11), as used in most recent studies $[23,24,26,31]$.

As illustrated in Fig. 1, an IMANET consists of a set of MTs that can communicate with each other using an ad hoc communication protocols (illustrated by the dashlines). An MT can move in any direction and make information search and access requests from anywhere in the covered area. Among the MTs, some of them can directly connect to the Internet, and thus serve as $\mathrm{APs}^{2}$ for the rest of MTs in the IMANET. An MT located out of the communication range of the AP has to access the Internet via relays through one of the APs. Thus, an AP is a gateway to the Internet and is assumed to have access to any information. An AP is located in a communication area and is connected to a database server. ${ }^{3}$

Although there is an approach [4] to integrate the 802.11 local area wireless networks and third-generation $(3 \mathrm{G})$ wide area wireless networks (e.g. CDMA 2000 and UMTS), we do not consider the issues of interoperability. Thus, we assume that an MT does not receive any data item directly through the $3 \mathrm{G}$ networks when it is out of

\footnotetext{
${ }^{2} \mathrm{An}$ AP here is a logical notation. An AP equipped with appropriate antennas can directly communicate with the Internet through wireless infrastructures including cellular base stations (BSs), Low Earth Orbit (LEO), or geostationary (GEO) satellites.

${ }^{3}$ From an MT's point of view, since an AP is transparent to a database server, we use the terms AP and database server (later in short, server) interchangeably.
} 
the communication range of an $\mathrm{AP}$, but receives indirectly by multi-hop relays.

A database may be attached to an AP, a fixed router or a database server. We assume that the database consists of a total $n$ data items $\left(D B_{\max }\right)$. A data item $(d)$ is the basic unit of an update or query operation. The database is only updated by the server, while a query is generated by the MTs for read-only requests. An MT can cache a limited number of data items since the size of the cache space in an MT is relatively small compared to the total number of data items in the database.

Depending on the cache invalidation strategy, a server can broadcast the list of $i d s$ of updated data items to the MTs to ensure data consistency, either synchronously (periodically) or asynchronously (non-periodically). When a server broadcasts ids, flooding technique can be used to ensure that every node receives the message. Since the flooding causes a heavy traffic, the multicast tree for broadcast can be used to reduce the message overhead. Thus, in this paper, we assume that the multicast tree is established and techniques proposed in $[7,36]$ can be used to setup and maintain the tree in IMANETS.

\section{A GPS-based connectivity estimation scheme}

In an IR-based cache invalidation scheme for cellular networks $[3,5,12,15,35,38]$, even if a query can be answered by a cached data item, an MT always waits for the next IR. Then it invalidates the cached data items accordingly, and answers the query if the cached copy is still valid. Otherwise, the MT sends a request to the server for a valid copy. Here, the basic assumption is that an MT is supposed to receive an IR regularly.

However, in an IMANET, an MT may not receive an IR due to link disconnections and time-varying network topologies. Without knowing whether an MT can receive an IR from the server directly or indirectly through multi-hop relays, it may wait for the next IR, which may not arrive at all. It implies that, unlike a cellular network, connectivity with the server is not always assured. Although connectivity can be restored by adjusting the MT's transmission power [14] or by modifying the MT's trajectory [21], we do not consider these techniques in this study for the sake of simplicity.

To address this issue, we propose a GPS-based connectivity estimation (GPSCE) scheme, which serves as a basic function for supporting cache invalidation mechanisms in IMANETS. In this scheme, by using the location information obtained by the GPS, an MT knows whether it is currently connected to a server. When an MT is unable to send (receive) a message to (from) a server, a query cannot be processed, because an MT neither can check the validity of a cached data item nor can receive a valid data item from a server. As long as the MT is aware of its status, it can decide what operation to follow, such as wait for an IR, send a request message, or deny a query. Thus, the
GPSCE scheme enables MTs to take the operation for verifying a query immediately.

Providing a location tracking capability in mobile devices is feasible due to the falling cost of GPS processors, low-power GPS receiver on board, and increasing number of potential applications such as an E-911 and a car's navigation system $[9,27]$. Since the Federal Communications Commission (FCC) mandates wireless service providers to supply high-precision location of mobile users in a lifethreatening situation (i.e., who make E-911 calls), GPS deployment in MT becomes popular. Implementation of low-power GPS receiver on board also makes its deployment in MT viable [33]. Thus, we assume that an MT is equipped with an on-board GPS receiver and is aware of its current location. Although other location positioning technologies [6,29] are also available, we do not consider those in this paper. Similar GPS-based techniques $[2,20]$ have been used for developing routing algorithms for MANETS. In this paper, for simplicity, we assume that the accuracy of GPS will be improved enough to ignore an error in the near future, e.g. NAVSTAR Global Positioning System has about 50 to $100 \mathrm{~m}$ of accuracy but the differential GPS has a few meters of accuracy [1]. Finally, since the accuracy of GPS may affect the performance, we will further discuss it as future work in Section 7.

\subsection{The proposed GPSCE scheme}

Using the GPS, we develop a connectivity estimation algorithm, called GPSCE. This algorithm estimates the maximum communication period of an MT, which is located a single-hop or multi-hops away from a server, based on the distance. Then, the MT is aware of its communication status to a server.

The algorithm works as follow: Suppose that an MT $i$ $\left(n_{i}\right)$ samples its location periodically. As shown in Fig. 2(a), the locations of $n_{i}$ at time $t$ and $t+\Delta t$ are $i(t)=\left(x_{1}, y_{1}\right)$ and $i(t+\Delta t)=\left(x_{2}, y_{2}\right)$, respectively. Based on these two locations, a vector of $n_{i}$ is given by

$\vec{i}=<x_{2}-x_{1}, y_{2}-y_{1}>$.

By using the vector $\vec{i}$, we can predict a new location of $n_{i}$ after time $t^{\prime}$ by $i\left(t^{\prime}\right)=\left(a_{i} \cdot t^{\prime}+x_{i}, b_{i} \cdot t^{\prime}+y_{\mathrm{i}}\right)$, where $a_{i}$ $\left(=x_{2}-x_{1}\right)$ and $b_{i}\left(=y_{2}-y_{1}\right)$ are the $x$ and $y$ vectors of $n_{i}$, respectively.
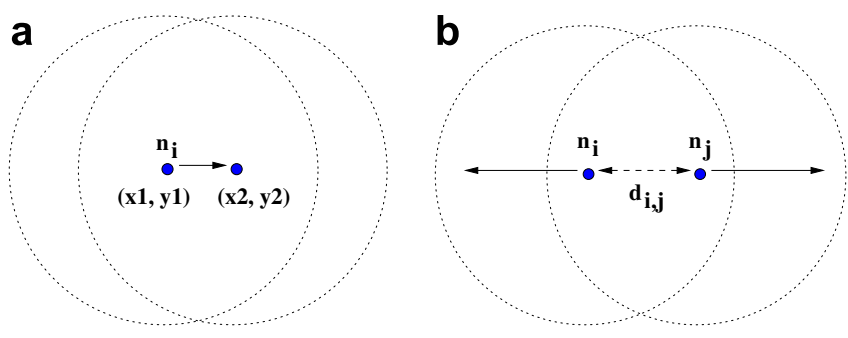

Fig. 2. A vector of $n_{i}$ in (a), and the distance between $n_{i}$ and $n_{j}$ in (b). 
Let us assume that the current locations, and the vectors of two MTs $n_{i}$ and $n_{j}$ are $i=\left(x_{i}, y_{i}\right)$ and $\left.\vec{i}=<a_{i}, b_{i}\right\rangle$, and $j=\left(x_{j}, y_{j}\right)$ and $\vec{j}=<a_{j}, b_{j}>$, respectively. In Fig. 2(b), if $n_{i}$ and $n_{j}$ move in opposite directions, they will be out of each other's communicate range. To estimate the period in which both $n_{i}$ and $n_{j}$ are located within each other's communication range, first we calculate the distance between them. The new locations of $n_{i}$ and $n_{j}$ after time $t^{\prime}$ are $i\left(t^{\prime}\right)=\left(a_{i} \cdot t^{\prime}+x_{i}, \quad b_{i} \cdot t^{\prime}+y_{i}\right) \quad$ and $\quad j\left(t^{\prime}\right)=\left(a_{j} \cdot t^{\prime}+x_{j}\right.$, $\left.b_{j} \cdot t^{\prime}+y_{j}\right)$. Thus, the distance between them, $d_{i, j}$, is given by

$d_{i, j}=\sqrt{\left(\left(a_{j}-a_{i}\right) \cdot t^{\prime}+\left(x_{j}-x_{i}\right)\right)^{2}+\left(\left(b_{j}-b_{i}\right) \cdot t^{\prime}+\left(y_{j}-y_{i}\right)\right)^{2}}$.

During communication, both $n_{i}$ and $n_{j}$ should be located within each other's communication range $(R)$. Here, we assume that all the MTs in a network use the same communication range, which is already known to them. Thus, $d_{i, j}$ should satisfy the following condition.

$d_{i, j} \leqslant R$.

Since $d_{i, j}$ is positive, we have $d_{i, j}^{2}-R^{2} \leqslant 0$. From this, we can estimate the maximum period $(P>0)$ in which both $n_{i}$ and $n_{j}$ are located within each other's communication range as

$$
P=\frac{-e+\sqrt{e^{2}-4 \cdot c \cdot h}}{2 \cdot c}
$$

where $c=\left(a_{j}-a_{i}\right)^{2}+\left(b_{j}-b_{i}\right)^{2}$,

$$
\begin{aligned}
& e=2 \cdot\left(\left(a_{j}-a_{i}\right) \cdot\left(x_{j}-x_{i}\right)+\right. \\
& \left.\left(b_{j}-b_{i}\right) \cdot\left(y_{j}-y_{i}\right)\right), \text { and } \\
& h=\left(x_{j}-x_{i}\right)^{2}+\left(y_{j}-y_{i}\right)^{2}-R^{2} .
\end{aligned}
$$

Based on the above equation, an MT $n_{i}$ that is a single-hop away from a server can obtain the $P_{i}$ to the server. Once $n_{i}$ knows the $P_{i}$, it can calculate the connection expire time $\left(T_{i}\right)$, the time when it is out of the communication range of a server. This is given by

$T_{i}=t_{\mathrm{cur}}+P_{i}$,

where $t_{\text {cur }}$ is the current time. For the MTs, which are multihops away from a server, let us assume that an $\mathrm{MT} n_{j}$ accesses the server by relaying a message through $n_{i}$ (refer to Fig. 4). Also, $T_{i}$ and $T_{j}$ are the connection expire times between the AP and $n_{i}$, and $n_{i}$ and $n_{j}$, respectively. If any one of these con- nections is broken, $n_{j}$ loses its connection to the server. Thus, we choose the minimum value of $T$, and $T_{j}$ is given as

$T_{j}=\operatorname{Min}\left(T_{i}, T_{j}\right)$.

Once $n_{j}$ calculates $T_{j}$, it can decide whether it is currently connected to a server. In this paper, the value of $T$ is used as an initial condition to proceed with a query for any cache invalidation strategy.

\subsection{A communication protocol for the GPSCE scheme}

In this subsection, we describe the communication protocol of the GPSCE scheme. When the connection expire

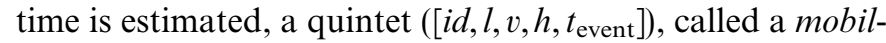
ity quintet $(Q)$, is attached to a message. Here $i d$ is the identification of a server or an MT. $l$ and $v$ are the current location and vector, respectively. $h$ is the number of hops from a server, and $t_{\text {event }}$ is the time when a message is sent. Each MTs keeps a status bit $(f)$ to indicate whether it is accessible to a server. Initially, all the MTs set $f$ to 0 .

We now describe three cases to estimate the communication period; (1) when an MT is single-hop away from a server, (2) multi-hop away from a server, and (3) when an MT changes its direction. First, we explain Case 1 and list the pseudo code for the single-hop protocol in Fig. 3. Note that if $n_{i}$ does not move $\left(v_{i}=0\right)$, $T_{i}$ is infinite. After setting $f_{i}$ to $1, n_{i}$ stops broadcasting the hello packet. To keep a seamless connection with a server, we proactively broadcast a hello packet before $t_{\text {cur }}$ becomes $T_{i}$. Otherwise, the MTs cannot response during the hello message interaction to estimate a new $T$, even though they are connected to a server. When $n_{i}$ receives a hello_ack packet, it repeats step 3. If $n_{i}$ does not receive a packet within a specified timeout period, $n_{i}$ sets $f_{i}$ to 0 and initiates to broadcast a hello packet periodically.

Next, we describe Case 2. Let us assume that an MT $n_{i}$ and the server are within each other's communication range $\left(f_{i}=1\right)$, as shown in Fig. 4 , and $n_{j}$ tries to decide whether it is accessible to the server. Since $n_{j}$ accesses the server through $n_{i}$, if $n_{i}$ is out of the communication range of the server, $n_{j}$ can no longer communicate with the server regardless of its connectivity with $n_{i}$. Also, $n_{j}$ may receive multiple hello_ack packets, but it chooses the packet in a

\section{Single-hop Protocol:}

1. When $f_{i}$ is $0, n_{i}$ broadcasts an one-hop hello packet to the adjacent MTs, periodically.

2. When a server, $r$, receives a hello packet, it replies a hello ack packet with a quintet, $Q_{r}=\left[i d_{r}, l_{r}, v_{r}, h_{r}, t_{\text {event }}\right]$ Here, $h_{r}$ is 0 .

3. When $n_{i}$ receives the hello ack packet, it calculates the $P_{i}$ based on the received $Q_{r}\left(l_{r}\right.$ and $\left.v_{r}\right)$, and its current location and vector by using Eqs. 2,3, and 4 . Then $n_{i}$ obtains $T_{i}$ from using Eq. 5, sets $f_{i}$ to 1 , and caches $Q_{r}$. Also $n_{i}$ sets $h_{i}$ to 1 by increasing $h_{r}$.

Fig. 3. The pseudo code of the single-hop protocol. 


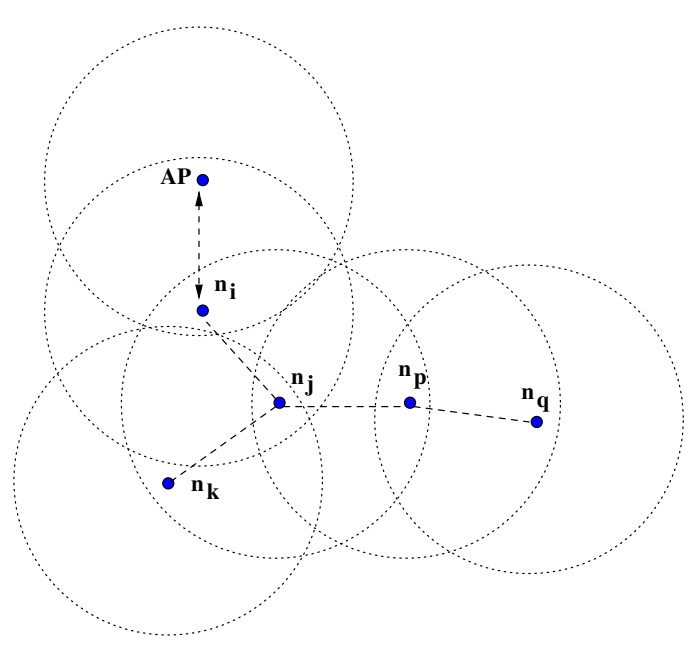

Fig. 4. A sample network topology.

first come first serve (FCFS) manner. The multi-hop protocol is summarized in Fig. 5.

Finally, we describe Case 3. When an MT is a single-hop away from a server, it recalculates $T$ based on the server's location and vector that are fixed values, and its new location and vector. When an MT is multi-hops away from a server, it should recalculate $T$ and broadcast a message to the MTs that access the server through it. Let us assume that $n_{i}, n_{j}$, $n_{k}, n_{p}$, and $n_{q}$ have connection with a server directly or indirectly through multi-hop relays (refer to Fig. 4). Assume that, $n_{j}$ changes its direction. When $n_{i}$ receives a loc update packet, it ignores the packet because any direction change does not affect $n_{i}$ 's connectivity to the server as long as it is in the communication range. The pseudo code for direction change protocol is given in Fig. 6.

An MT may also determine its connectivity to a server using an alternative technique. For example, an MT keeps a list of one-hop neighbor by exchanging an one-hop hello message without using GPS information. In this approach, the MT should keep sending a message periodically to check whether it has access to the server, even though it is in the communication range of a server. Thus, this approach would generate high message traffic. In our scheme, we do not generate a hello packet if $f$ is 1 .

\section{Cache invalidation strategies for IMANETs}

In this section, we first discuss a modified simple search (MSS) algorithm for implementing the invalidation policy. The MSS algorithm finds queried data items efficiently in IMANETS. Then we present the four cache invalidation schemes. An aggregate cache-based on demand (ACOD) scheme is proposed based on the GPSCE scheme for IMANETS. Also, we modify two different cache invalidation strategies, proposed for cellular networks, to work in IMANETS. These are a modified timestamp (MTS) scheme and an MTS with updated invalidation report (MTS + UIR) scheme.

\subsection{A modified simple search algorithm}

In an IMANET, the data items from a server may be cached in some of the MTs. Thus, a search scheme needs

Multi-hop Protocol:

1. When $f_{j}$ is $0, n_{j}$ broadcasts a hello packet.

2. When $n_{i}$ receives a hello packet from $n_{j}$, it checks the $f_{i}$ value to decide whether it can reply. Since $f_{i}$ is $1, n_{i}$ replies a hello ack packet including both $Q_{i}$ and $T_{i}$ to $n_{j}$. If $f_{i}$ is 0 , then $n_{i}$ ignores the hello packet.

3. When $n_{j}$ receives a hello ack packet from $n_{i}$, it calculates $T_{j}$ in the same manner described in the single-hop protocol. Then $n_{i}$ finds the minimum value of $T_{j}$ by using Eq. $6\left(T_{j}=\operatorname{Min}\left(T_{i}, T_{j}\right)\right)$, and caches the hello ack packet sender's quintet $\left(Q_{i}\right)$, sets $f_{j}$ to 1 , and stops broadcasting the hello packet.

Fig. 5. The pseudo code of the multi-hop protocol.

\section{Direction Change Protocol:}

1. When $n_{j}$ changes its direction, it updates its position vector and recalculates $T_{j}$. To recalculate $T_{j}, n_{j}$ requires the location of $n_{i}$ that replied a hello ack packet before. For this, we utilize the $Q_{i}$ cached in $n_{j}$ to predict the location of $n_{i}$. Since $n_{j}$ knows the vector and location of $n_{i}$ at $t_{\text {event }}$, it can estimate the current location of $n_{i}$ at $t_{\text {cur }}\left(i\left(t_{\text {cur }}\right)\right.$ $=\left(v_{i, x} \cdot\left(t_{\text {cur }}-t_{\text {event }}\right)+l_{i, x}, v_{i, y} \cdot\left(t_{c u r}-t_{\text {event }}\right)+l_{i, y}\right)$, where $l_{i, x}$ and $l_{i, y}$, and $v_{i, x}$ and $v_{i, y}$ are the location and vector of $n_{i}$, respectively.). Based on the new location of $n_{i}, n_{j}$ can recalculate $T_{j}$ by using Eqs. 2, 3, and 4 . Then $n_{j}$ broadcasts an one-hop loc update packet, attached with the updated $Q_{j}$ to the MTs that rely on accessing the server through $n_{j}$ (e.g. $n_{k}, n_{p}$, and $n_{q}$ ).

2. When $n_{k}$ receives a loc update packet from $n_{j}$, it compares the $i d$ of the packet sender with the $i d$ cached in the quintet. If it matches, $n_{k}$ recalculates $T_{k}$ based on the received quintet by using Eqs. 2, 3, and 4. Then $n_{k}$ chooses the minimum value $\left(T_{k}=\operatorname{Min}\left(T_{j}, T_{k}\right)\right)$, and caches the received quintet. Similarly, $n_{p}$ and $n_{q}$ update their $T \mathrm{~s}$. If the $i d$ does not match, the loc update packet is ignored.

Fig. 6. The pseudo code of the direction change protocol. 
to be deployed for finding a data item whether from the server or from an MT. In this paper, for efficient search of queried data items, we propose a modified simple search (MSS) algorithm that is an extended version of a simple search $(S S)$ scheme [23], in which the cache update is not considered. To reduce the query delay and number of communication messages, we use an aggregate cache concept in

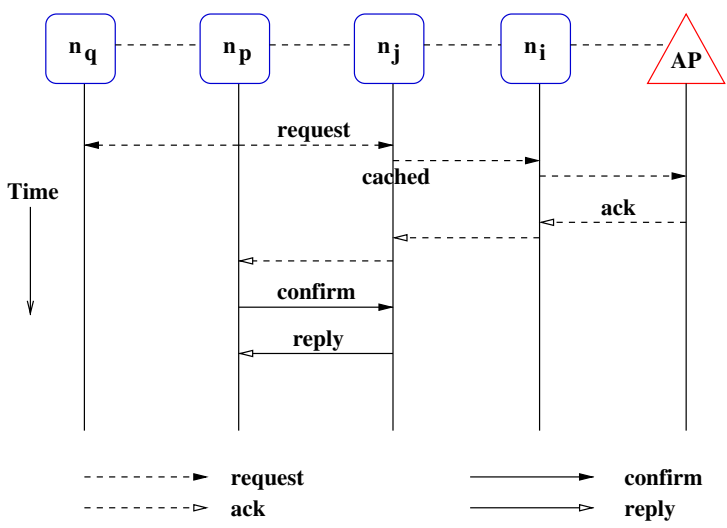

Fig. 7. A modified simple search (MSS) algorithm for querying data items in the IMANET. Let us assume that an MT $\left(n_{p}\right)$ sends a request for a data item, and another MT $n_{j}$ receives the request. $n_{j}$ has a copy of the queried data item in its local cache. $n_{j}$ sends a request to the server to check the validity of the data item. When the server receives the request, it verifies the status of the queried data item, and replies an ack packet. Then $n_{p}$ sends a confirm packet to $n_{j}$, and $n_{j}$ attaches the queried data item to the reply packet. Here, the dotted line between the MTs or an MT and an AP represents that they are located within the communication range. which, a data item can be received from either the local cache of the MTs or from a server.

In the original SS scheme, when a query is generated, an MT broadcasts a request to all of the adjacent MTs. If an MT receives the request and has the data item in its local cache, it sends a reply to the requester to acknowledge that it has the data item. Otherwise it forwards the request to the neighbors. Thus, a request may be flooded in the network until it is acknowledged by a server and/or some MTs, which have cached copies of the queried data item. The sender thus can receive a data item from the closest MT or server.

For implementing a cache invalidation scheme, when a query is generated which can be answered by a cached data item in an MT, the MT sends a message to a server to verify whether the cached data item is a valid copy. If the data item is invalid, the MT receives the valid copy from the server and uses it. Basically, the SS algorithm broadcasts a request to all neighboring $\mathrm{MTs}$ for a response. Since the cache update is only received from a server, in the MSS scheme, the broadcasted requests are sent only to the MTs, which are along the closer path to the server. This is different from the SS scheme, where a request is broadcasted to all neighboring MTs irrespective of the direction. The MSS scheme is thus a directed broadcast, and the direction can be obtained from the hop count, encapsulated in a packet. The MSS scheme can be implemented using any existing routing protocol. The detailed steps

\section{Modified Simple Search Protocol:}

1. When a query is generated, $n_{p}$ checks its local cache. If the queried data item is available, $n_{p}$ immediately broadcasts a request packet to a server. A request packet contains the requester's id $(i d)$, id of the queried data item $\left(d_{s}\right.$, where $\left.0 \leq s<D B_{\max }\right)$, the most recently updated timestamp of $d_{s}\left(t_{s}\right)$, and number of hop $(h)$ from the server.

2. When $n_{j}$ receives the request, it compares the current number of hops from the server with $h$ attached in the packet. Since $n_{j}$ is closer to the server, it checks its local cache. If the queried data item is cached, $n_{j}$ compares its updated timestamp with $t_{s}$ attached in the packet. By updating $t_{s}$ with the most recent value and attaching its id, $n_{j}$ indicates that it has a cached copy. Then $n_{j}$ forwards the request to the server to check the validity of the data item.

3. When $n_{q}$ receives the request packet, it compares the current number of hops from the server with $h$ attached in the packet. Since $n_{q}$ is further away from the server, it does not forward the packet, and discards it. Thus, the MSS algorithm attempts to find the shortest path to the server.

4. When a server receives the request packet, it compares the updated timestamp of data item stored in the database with $t_{s}$, attached in a packet. The server replies with an ack packet attached with the response (either the updated data item or a valid bit telling that the data item is valid in the cache) to $n_{j}$, which subsequently sends it to the requester

5. When $n_{p}$ receives an ack packet, it uses the cached data item to answer the query, if it has a valid copy. Otherwise, it sends a confirm packet for a valid data item to $n_{j}$. Here, $n_{p}$ may receive multiple ack packets, but it chooses the packet which arrives first. Since the packets are forwarded through different paths, $n_{j}$ selects the path for a confirm packet based on the first receipt of the ack packet, and discards rest of the ack packets.

6. When $n_{j}$ receives a confirm packet, it sends a reply packet to the confirm packet sender using the known route with the cached data item, which is already verified by a server. When a server receives a confirm packet, it attache the valid data item to the reply packet.

Fig. 8. The modified simple search protocol. 
are described in Fig. 8. Let us assume that an MT $n_{p}$ generates a request for a data item as shown in Fig. 7.

When an MT forwards a request packet, the id of the MT is appended in the packet to keep the route information. Also, when an MT receives multiple copies of a request packet, due to broadcasting, it only processes one of them and discards the rest. For an ack, confirm, or reply packet, the MT also checks if its id is included in the path, which is appended to the packet. Since these packets are supposed to travel only along the assigned path that is established by the request packet, if the MT's id is not included in the path, the packet is discarded. When the server receives a request packet, it does not send the data item immediately, but sends an ack because other MTs, which are located closer to the sender might cache the valid data item. This helps in reducing network congestion and bandwidth consumption by multiple replies for a single query.

\subsection{An aggregate cache based on demand ( $A C O D$ ) scheme}

In contrast to the prior push-based schemes designed for cellular networks $[3,5,12,15,17,35,38]$, where the server periodically/non-periodically broadcasts a message, we propose a pull-based approach. In this approach, whenever a query is generated, an MT sends a message to a server to verify a cached data item before it is used for answering the query.

The basic mechanism is described as follows. When a query is generated, an MT checks its $f$ value, which is obtained by invoking GPSCE. If $f$ is 0 , which implies that the MT cannot access a server, the query fails. If $f$ is 1 , the MT checks its local cache, and broadcasts a request packet by using the proposed MSS algorithm. When an MT receives the request, it checks its $f$ value. If $f$ is 1 , the MT checks its local cache, and forwards the packet to the MTs closer to the server. Thus, the sender receives either an acknowledgment from a server to indicate that its cached data item is valid or the queried data item from a server or an adjacent MT, who has the valid data item. The pseudo code for the ACOD scheme is given in Fig. 9.

\subsection{A modified timestamp (MTS) scheme}

In the TS scheme [3] proposed for cellular networks, a server periodically broadcasts an IR that carries the current timestamp $\left(t_{\text {cur }}\right)$ and a list of tuples $\left(d_{s}, t_{s}\right)$ such that $t_{s}>t_{\text {cur }}-w \cdot L$, where $d_{s}$ is a data item id and $t_{s}$ is the most recently updated timestamp of $d_{s}\left(0 \leqslant s<D B_{\max }\right)$. Here, $w$ is an invalidation broadcast window and $L$ is the broadcast interval. If an MT is in the active mode, it receives the IR and invalidates its cached data items accordingly. If an MT is disconnected or is in the sleep mode for more than $w \cdot L$, it invalidates the entire cached data items because it cannot decide which data items are valid. In this paper, we do not consider the long disconnection problem for the sake of simplicity.

We modify the MTS scheme for IMANETS. The basic mechanism is described as follows. When a query is generated, an MT checks its $f$ value. If $f$ is 1 , then the MT either waits for the next IR, or broadcasts a request packet by

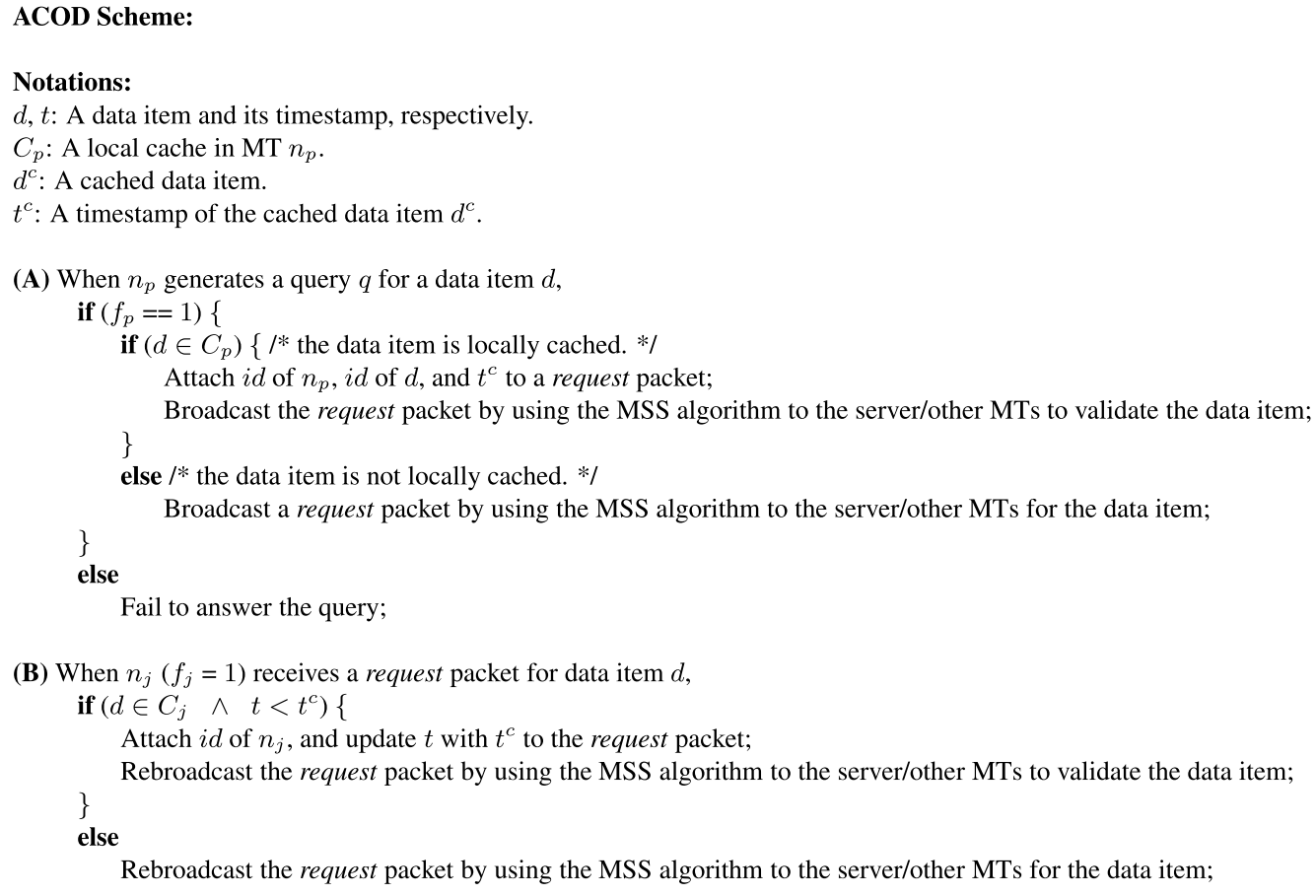

(B) When $n_{j}\left(f_{j}=1\right)$ receives a request packet for data item $d$,

Fig. 9. The pseudo code of the ACOD scheme. 


\section{MTS Scheme:}

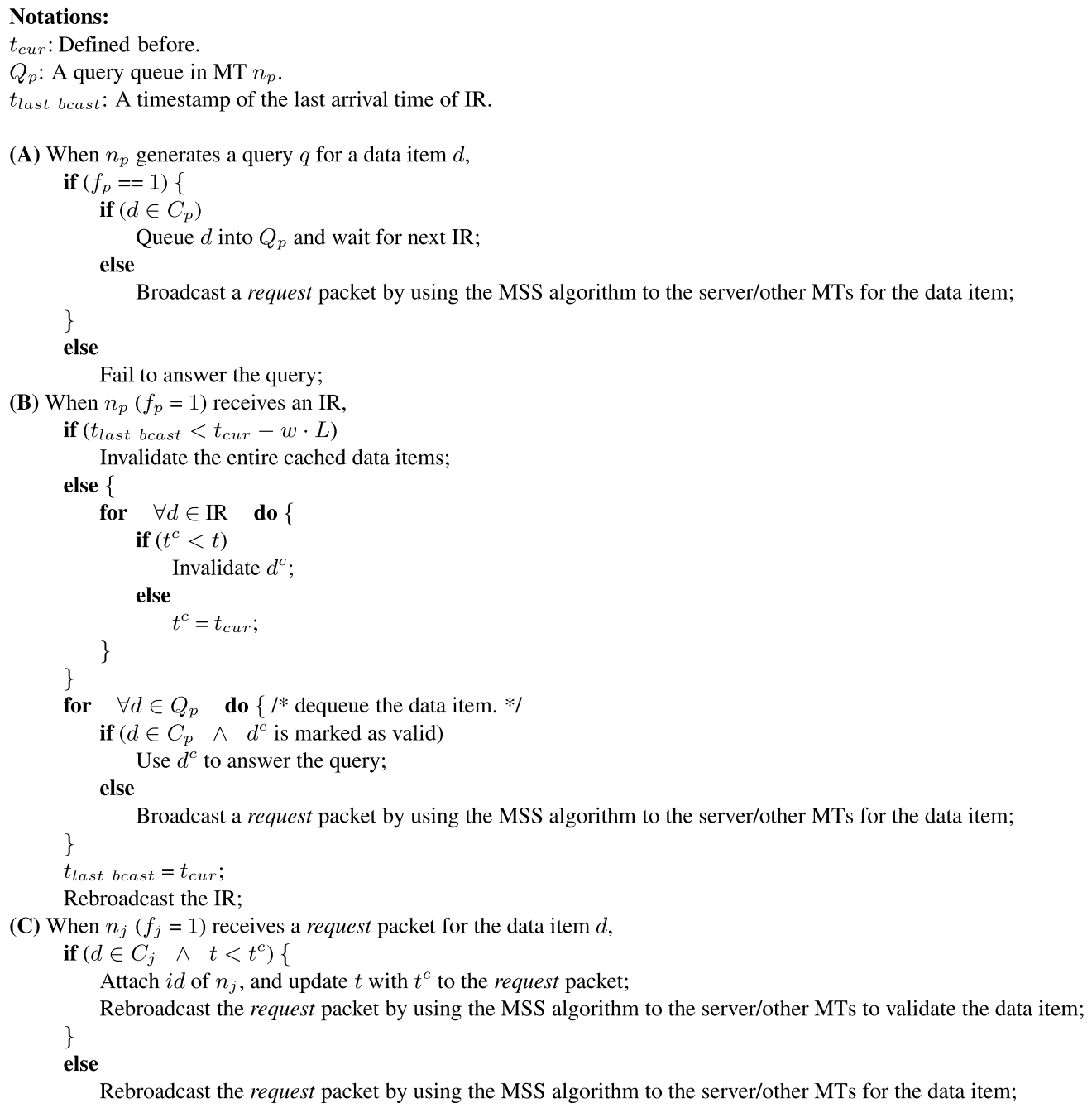

Fig. 10. The pseudo code of the MTS scheme.

using the MSS algorithm. After the MT receives the IR, it invalidates the cached data items accordingly, and forwards the IR to adjacent MTs. If a queried data item is still valid, the MT uses it to answer the query. Otherwise, the MT broadcasts a request packet. When an MT receives a request packet, it forwards the packet to a server rather than waiting for the next IR. Although an MT can reply an ack packet while waiting for the next IR, it forwards the packet to a server. Otherwise, the query delay may increase. Here, we use a hop limit for an IR to prevent flooding of the IR packets over the network. An MT does not rebroadcast an IR, when it already has received the same IR, or the number of forwarded hops of the IR exceeds the hop limits. We use a hop limit for a IR packet to prevent large number of floating packets from the network. The pseudo code for the MTS scheme is shown in Fig. 10.

A major drawback of the MTS scheme is the unavoidable query delay due to periodic broadcast of IRs.

\subsection{An MTS with updated invalidation report (MTS + UIR) scheme}

This scheme is the same as the MTS protocol except that an updated invalidation report (UIR), which is partially derived from [5], is added. An UIR packet contains a timestamp of the last broadcasted IR from a server $\left(t_{\text {last_bcast }}\right)$, and a list of tuples $\left(d_{s}, t_{s}\right)$ that have been updated after the last IR has been broadcasted, where $t_{s}>t_{\text {last_bcast. }}$. The server inserts a number of UIRs into the IR intervals. Thus, an MT can process a query after receiving either an IR or an UIR. In this paper, we use 4 UIR replicates within each IR interval.

The MTS + UIR scheme follows the same procedure as the MTS scheme except followings. When an MT receives an UIR, it compares $t_{\text {last_bcast }}^{c}$ saved in the local cache with $t_{\text {last_bcast }}$ received from the UIR. If $t_{\text {last_bcast }}^{c}>\left(t_{\text {last_bcast }}-L\right)$ is true, the MT invalidates the cached data items accordingly, and answers the query or sends a request packet 
ASYNC Scheme:

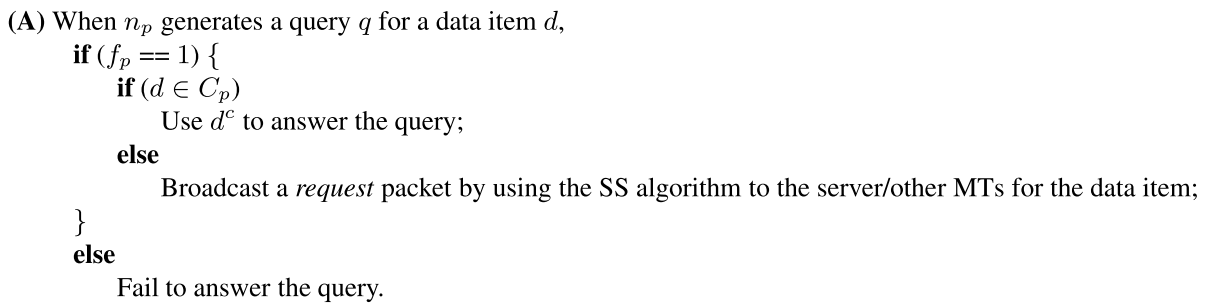

(B) When $n_{p}\left(f_{p}=1\right)$ receives an IR, Invalidate cached data items, accordingly; Rebroadcast the IR;

(C) When $n_{j}\left(f_{j}=1\right)$ receives a request packet that requests $d$, if $\left(d \in C_{j}\right)$

Reply an ack packet to the request packet sender by using the SS algorithm; else

Rebroadcast the request packet by using the SS algorithm to the server/other MTs for the data item;

Fig. 11. The pseudo code of the ASYNC scheme.

using the MSS algorithm. Otherwise, the MT ignores an UIR and waits for the next IR.

While this scheme reduces the query delay compared to the MTS scheme, it has higher message overhead than that of the MTS scheme due to additional UIR broadcasts.

\subsection{Discussion}

In IR-based approach, due to server's periodic IR broadcast, the MT can save a battery power by switching an operation mode into a doze mode. The MT should wait for next IR before answering a query and thus, there is an inevitable delay. However, in some situations such as in the battle-field, it is critical for all MTs to be updated to execute a military operation. In this case, reducing power consumption is not an issue. Thus, we consider a scheme suitable for such a delay-sensitive application, called asynchronous ( $A S Y N C)$.

In this scheme, unlike the periodically broadcasted IR, a server broadcasts an IR whenever a data item is updated. If an MT has access to a server, it can decide the validity of current cached data items based on the broadcasted IR. Thus, when a query is generated, which can be answered by a cached data item, an MT does not contact the server for checking its validity. This approach is different from a prior scheme [17] proposed for cellular networks, where a stateful server broadcasts an IR asynchronously to the MTs that have the cached copy of the data item. However, the stateful approach is not feasible in IMANETS because of the high message overhead to maintain such information.

The mechanism of this scheme is described as follows. When a query is generated, an MT checks its $f$ value. If $f$ is 1 and the queried data item is found in its local cache, the MT uses the cached data item to answer the query. However, if $f$ is 1 and the queried data item is not available in the local cache, the MT broadcasts a request packet by using the SS algorithm. Since an MT can response to the request, the ASYNC scheme uses the SS algorithm that searches the queried data item in any direction for better performance. Thus, the MT eventually receives the queried data item from a server and/or some closer MTs.

When an MT is disconnected from a server, it can neither receive an IR nor answer a query. Since an IR is non-periodically broadcasted, an MT cannot ensure validity of a cached data item without connection with a server. Thus, when an MT is reconnected to the server, it sends a cache update packet including a list of tuple $\left(d_{s}, t_{s}\right)$ of all cached data items to a server for validation. When the server receives the packet, it replies a cache update_reply packet including the data items that have been updated. When an MT receives the cache_update reply packet, it updates its cache accordingly. The pseudo code for the ASYNC scheme is given in Fig. 11.

In this paper, due to different goal, we do not compare the ASYNC scheme with other cache invalidation strategies, unless otherwise specified.

\section{Performance evaluation}

\subsection{Simulation testbed}

We use $n s-2$, a discrete event network simulator with the CMU's wireless extension, to conduct the experiments. The radio model simulates Lucent's Technologies WaveLAN [34] with a nominal bit rate of $2 \mathrm{Mbps}$ and a nominal range of $250 \mathrm{~m}$. The radio propagation model is based on the free-space model [28]. For the link layer, the IEEE 802.11 MAC protocol and the distributed coordination function (DCF) are used.

To examine the proposed idea, we use a $2000 \times 750 \mathrm{~m}$ rectangular area. We assume that an AP is located in the center of an area. The MTs are randomly located in the network. The speed $(s)$ of an MT is uniformly distributed in the range $(0.0<s \leqslant 2.0 \mathrm{~m} / \mathrm{s})$. The random waypoint mobility model, developed in [16], is used to simulate 
Table 1

Simulation parameters

\begin{tabular}{ll}
\hline Parameter & Values \\
\hline Network size (m) & $2000 \times 750$ \\
Number of MTs & 50 \\
Number of APs & 1 \\
Database size (items) & 1000 \\
Cache size (items/MT) & $10-100$ \\
Data item size (KByte) & 10 \\
Mean query interval time (s) & $5-200$ \\
Mean update interval time (s) & $10-1000$ \\
Hot data items & $1-50$ \\
Cold data items & Remainder of DB \\
Hot data item update prob. & 0.8 \\
Broadcast interval (s) & 20 \\
Broadcast window $(w)$ & 10 intervals \\
Pause time (s) & 100
\end{tabular}

mobility here. With this approach, an MT travels toward a randomly selected destination in the network. After the MT arrives at the destination, it chooses a rest period (pause time) from a Uniform distribution. After the rest period, the MT travels towards another randomly selected destination, repetitively.

The query arrival pattern follows the Poisson distribution with a rate of $\lambda$. The update inter arrival time to the database is assumed to be exponentially distributed. The entire data items in the database are classified into two subsets: cold and hot data. We assume $80 \%$ of the update requests are for hot data items, and an update request is uniformly distributed within the hot and cold subsets. The least frequently used (LRU) cache replacement policy is applied to the MT's local cache. To model the data item access pattern, we use the Zipf distribution [41] which is often used to model a skewed access pattern [5,13], where $\theta$ is the access skewness coefficient. Setting $\theta=0$ corresponds to the Uniform distribution. Here, we set $\theta$ to 0.9 . The important simulation parameters are summarized in Table 1.

\subsection{Simulation metric}

In this section, we evaluate five performance parameters: successful query rate $\left(M_{\text {suc }}\right)$, average query delay $\left(M_{\text {delay }}\right)$, average number of hop $\left(M_{\text {hop }}\right)$, cache hit ratio $\left(h_{\text {all }}\right)$ includ- ing local cache hit and remote cache hit, and average number of message $\left(M_{\mathrm{msg}}\right)$ for request.

The successful query rate, $M_{\text {suc }}$, is used to measure the accessibility of valid data items of the MTs in the IMANET. If $r_{\text {total }}$ and $r_{\text {suc }}$ denote the total number of queries and the number of successfully processed queries, then $M_{\text {suc }}$ is defined as,

$M_{\text {suc }}=\frac{r_{\text {suc }}}{r_{\text {total }}} \times 100 \%$.

The average query delay, $M_{\text {delay }}$, represents the average time to answer a query. If $q_{r}$ denotes the delay for a successfully processed query $r$, then $M_{\text {delay }}$ is expressed as,

$M_{\text {delay }}=\frac{\sum_{r \in r_{\text {suc }}} q_{r}}{r_{\text {suc }}}$.

The average number of hops, $M_{\mathrm{hop}}$, is the distance to a server or an MT to check the validity of a data item. If a query is satisfied by a cached data item in the local cache, then $M_{\text {hop }}$ is 0 . If $q_{h}$ denotes the number of hop for a successfully processed query $r$, then $M_{\text {hop }}$ is expressed as,

$M_{\text {hop }}=\frac{\sum_{r \in r_{\text {suc }}} q_{h}}{r_{\text {suc }}}$.

The hit ratio $h$ is used to evaluate the efficiency of the aggregate cache in our scheme. The remote cache hit is the ratio that a query is satisfied in other MTs' local cache. If $n_{\text {local }}$ and $n_{\text {remote }}$ denote the number of local hits and number of remote hits, respectively, then $h_{\text {local }}, h_{\text {remote }}$, and $h_{\text {all }}$ are expressed as:

$$
\begin{aligned}
h_{\text {local }} & =\frac{n_{\text {local }}}{n_{\text {local }}+n_{\text {remote }}} \times 100 \%, \\
h_{\text {remote }} & =\frac{n_{\text {remote }}}{n_{\text {local }}+n_{\text {remote }}} \times 100 \%, \\
h_{\text {all }} & =\frac{n_{\text {local }}+n_{\text {remote }}}{r_{\text {suc }}} \times 100 \% .
\end{aligned}
$$

Finally, the average number of messages, $M_{\mathrm{msg}}$, is counted to measure the overhead of an algorithm. Let $n_{\text {bcast }}, n_{\text {sent }}$, and $n_{\text {forward }}$ denote the total number of broadcasts, sent, and forwarded packets, respectively. Then $M_{\mathrm{msg}}$ is expressed as,
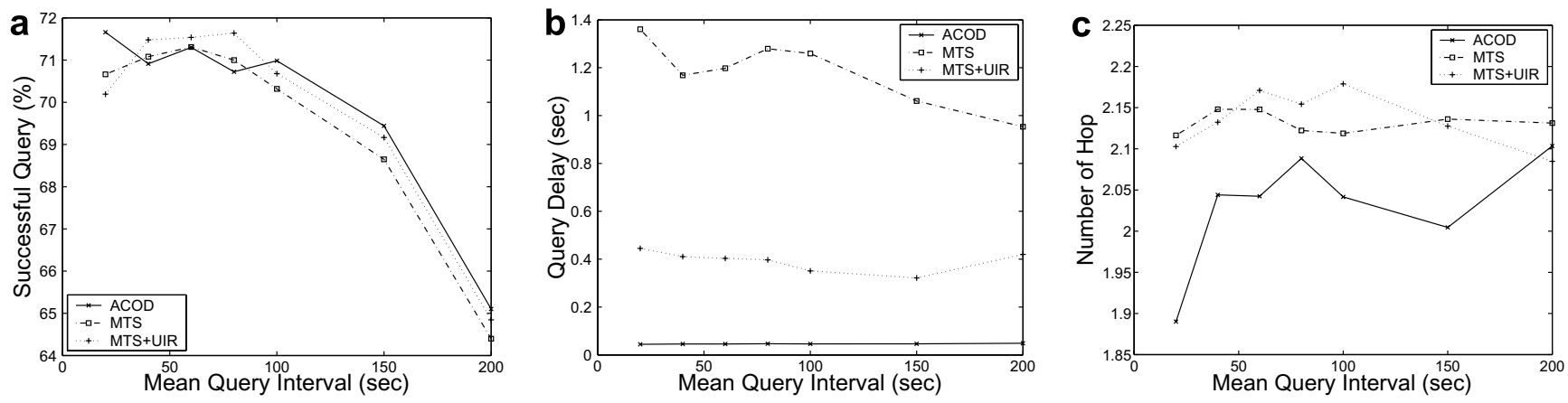

Fig. 12. (a) Successful query, (b) query latency, (c) the number of hops as a function of mean query interval $\left(T_{\text {update }}=100\right.$, and $\left.C_{\text {size }}=10\right)$. 
$M_{\mathrm{msg}}=\frac{n_{\text {bcast }}+n_{\text {sent }}+n_{\text {forward }}}{r_{\text {total }}}$

\subsection{Simulation results}

In this section, we evaluate the impact of query interval ( $\left.T_{\text {query }}\right)$, cache update interval $\left(T_{\text {update }}\right)$, size of the aggregate cache, and the search algorithm on the cache invalidation strategies. Also, the overhead of cache invalidation strategies is examined.

\subsubsection{Impact of query interval}

First, we evaluate the $M_{\text {suc }}, M_{\text {delay }}$, and $M_{\text {hop }}$ of the cache invalidation strategies as a function of $T_{\text {query. }}$. In Fig. 12(a), as the query interval increases, the percent of successful query decreases. Since all the cache invalidation strategies answer a query after either verification of a cached data item or arrival of an IR, they show almost similar performance under the same query interval and network topology. Fig. 12(b) shows the impact of $T_{\text {query }}$ on the query delay. The ACOD scheme shows the lowest $M_{\text {delay }}$, followed by the MTS + UIR and MTS schemes. The MTS scheme shows the longest $M_{\text {delay }}$, due to the long IR broadcast interval. In the MTS + UIR scheme, $M_{\text {delay }}$ is reduced compared to that of MTS due to UIRs, which arrive during an IR broadcast interval. Fig. 12(c) shows the impact of $T_{\text {query }}$ on the number of hops. The ACOD scheme shows slightly lower $M_{\text {hop }}$ than that of the MTS and MTS + UIR schemes. Since a data item can be received from an intermediate $\mathrm{MT}$, the number of hops is less compared to the IR-based scheme.

\subsubsection{Impact of update interval}

Next, we examine the impact of $T_{\text {update }}$ on $M_{\text {suc }}, M_{\text {delay, }}$, and $M_{\text {hop. }}$ In MTS and MTS + UIR schemes, from Fig. 13(a), $M_{\text {suc }}$ increases as the update interval increases. However, the ACOD scheme shows little change of $M_{\text {suc }}$ over the entire update intervals, because it checks validity of a cached data item or receives a queried data item from the server, and thus, its performance is not affected by $T_{\text {update }}$ much. In Fig. 13(b), the ACOD scheme shows the lowest $M_{\text {delay }}$ over the entire update intervals, followed by the MTS + UIR and MTS schemes. As the update interval increases, the $M_{\text {delay }}$ of both MTS and MTS + UIR schemes is increased. Since less number of cached data items are updated at a server, an MT finds more number of cached data items marked as valid. Thus, in the MTS and MTS + UIR schemes, the MT waits for the next IR or UIR arrival for validation frequently, and query delay increases. The MTS scheme has the longest $M_{\text {delay }}$, because it has longer interval to receive the next IR than that of the MTS + UIR scheme. In Fig. 13(c), as the update interval increases, $M_{\text {hop }}$ of all the schemes is proportionally reduced, because more number of cached
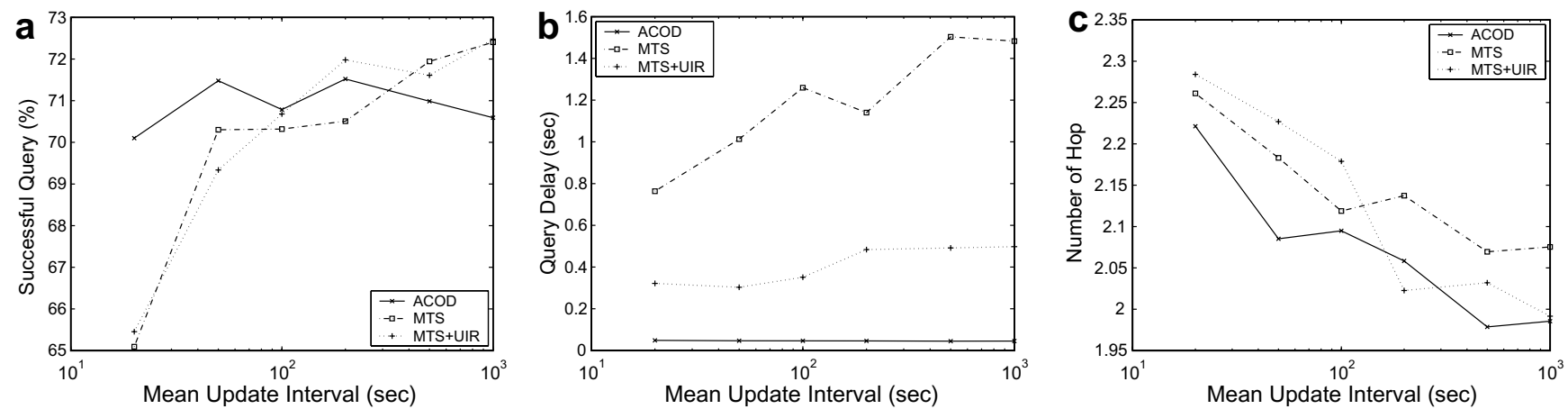

Fig. 13. (a) Successful query, (b) query delay, (c) number of hops as a function of mean update interval $\left(T_{\text {query }}=100\right.$, and $\left.C_{\text {size }}=10\right)$.
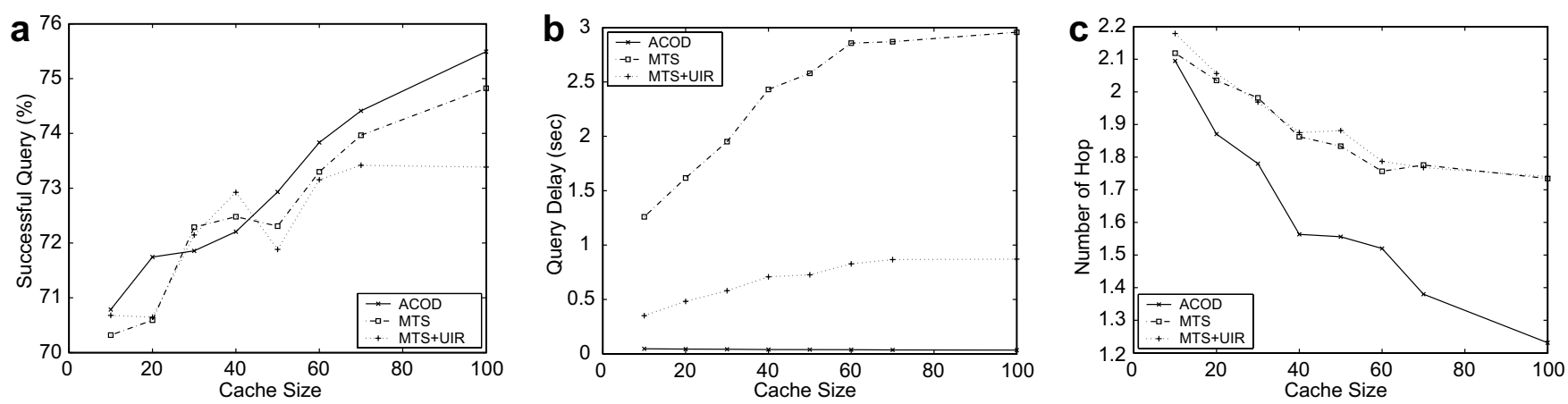

Fig. 14. (a) Successful query, (b) query delay, (c) number of hops as a function of cache size $\left(T_{\text {query }}=100\right.$, and $\left.T_{\text {update }}=100\right)$. 

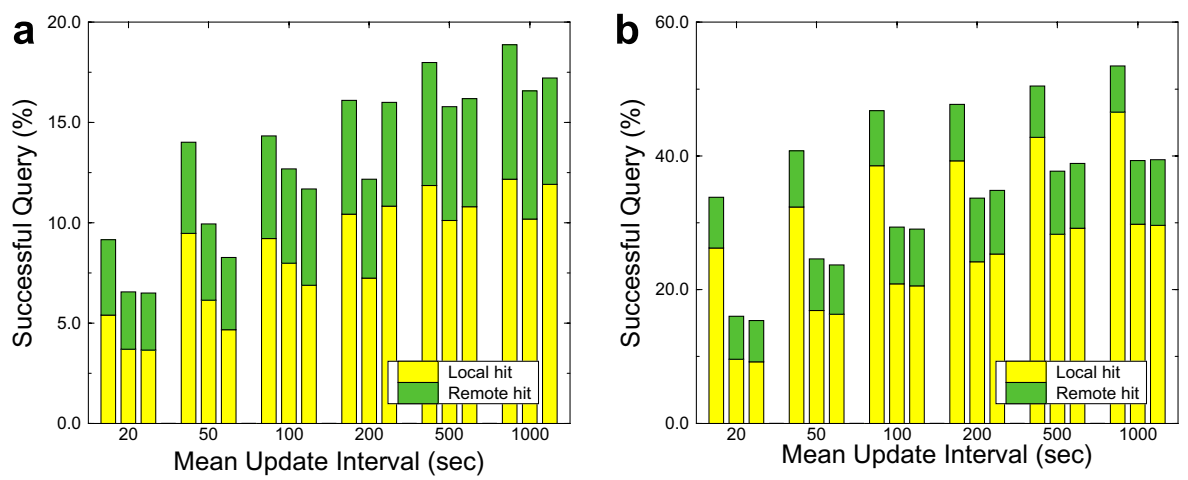

Fig. 15. Successful query in terms of average cache hit ratios is shown against mean update interval $\left(T_{\text {query }}=100\right.$, and $C_{\text {size }}=10$ (a) and $C_{\text {size }}=100$ (b)). The ACOD, MTS, and MTS + UIR schemes are plotted from left to right.

data items are valid, and thus an MT receives more queried data items from MTs rather than from a server.

\subsubsection{Impact of aggregate cache}

We observe the impact of aggregate cache on $M_{\text {suc }}, M_{\text {delay }}$, and $M_{\text {hop }}$ by varying the cache size. In Fig. 14(a), as the cache size increases, $M_{\text {suc }}$ of all the schemes is increased. Because more number of queried data items are answered from the local cache or remote caches. In Fig. 14(b), the MTS scheme shows the longest $M_{\text {delay, }}$ followed by the MTS + UIR and ACOD schemes. With a larger cache, as more number of data items are found in the cache, an MT waits for the next IR for validation. The MTS + UIR scheme reduces the $M_{\text {delay }}$, because an UIR arrives during the IR broadcast interval. In Fig. 14(c), as the cache size increases, the ACOD scheme results in the minimum hop count $\left(M_{\mathrm{hop}}\right)$. Since more data items can be received from an intermediate MT, the number of hops is much smaller than that of the IR-based scheme.

In Fig. 15, we plot $M_{\text {suc }}$ contributed due to only cache hits including local and remote hits. As the $T_{\text {update }}$ increases, more cached data items remain valid, and thus, the cache hits of all the schemes increase. As we expect, from Figs. 15(a) and (b), the cache hits increase as the $C_{\text {size }}$ increases. The ACOD scheme shows higher $M_{\text {suc }}$ than that of the MTS and MTS + UIR schemes, because it actively searches a queried data item during validation of a cached data item. In summary, the aggregate cache increases the cache hit, and thus, overall $M_{\text {suc }}$ increases.

\subsubsection{Impact of search algorithm}

We compare the proposed MSS algorithm with the SS algorithm, and evaluate their performances in terms of $M_{\text {msg, }}, M_{\text {suc }}$, and $M_{\text {delay }}$. Fig. 16 shows the impact of MSS and SS algorithms on $M_{\mathrm{msg}}, M_{\text {suc }}$, and $M_{\text {delay }}$ for different cache invalidation strategies. In Fig. 16(a), when the MSS algorithm is used, $M_{\mathrm{msg}}$ is reduced for all the cache invalidation strategies. In the MSS algorithm, unlike the SS algorithm, a request packet is broadcasted toward the MTs, which are located along the path/closer to the server. Thus, the MSS algorithm eliminates unnecessarily broadcasted messages. In Figs. 16(b) and (c), since the SS algorithm generates more messages over the network, it may cause traffic congestion and larger message delay. The MSS algorithm achieves higher $M_{\text {suc }}$ and lower $M_{\text {delay }}$ than that of the SS algorithm for all the cache invalidation strategies. In summary, the proposed MSS algorithm reduces the number of messages
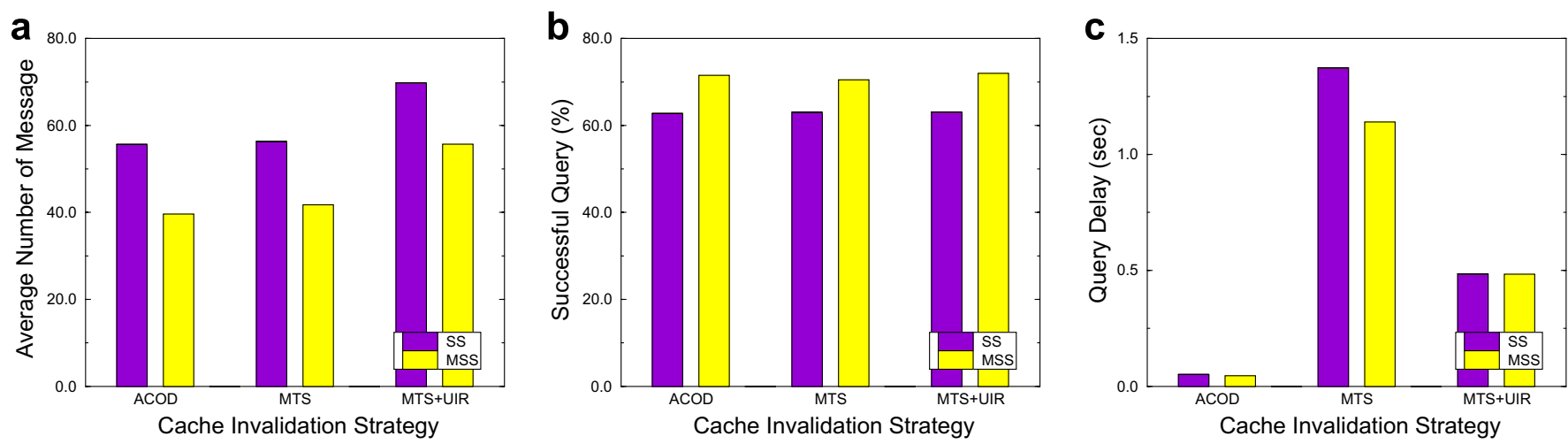

Fig. 16. (a) Average number of message, (b) successful query, and (c) query delay with different search algorithms are shown against different cache invalidation strategies $\left(T_{\text {query }}=100, T_{\text {update }}=200\right.$, and $\left.C_{\text {size }}=10\right)$. 


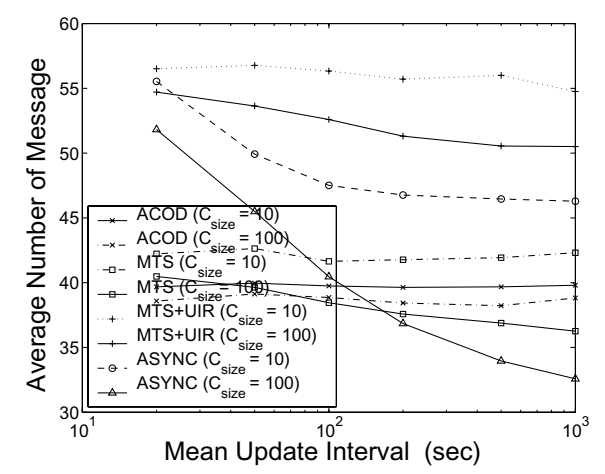

Fig. 17. Average number of messages as a function of mean update interval $\left(T_{\text {query }}=100\right.$, and $C_{\text {size }}=10$ or 100$)$.

and increases communication performance, and thus, it is suitable for implementing cache invalidation strategies for IMANETS.

\subsubsection{Message overhead}

Finally, we evaluate the overhead of each cache invalidation strategy in terms of average number of messages, including broadcast, sent, and forwarded messages from the server and MTs. Fig. 17 shows the impact of update interval on $M_{\text {msg }}$ with different cache sizes. The MTS + UIR scheme shows the highest $M_{\mathrm{msg}}$ because both IR and UIR are periodically broadcasted over the network, regardless of the update interval. When the update interval is small $\left(T_{\text {update }}=20\right)$, the ASYNC scheme has the high $M_{\mathrm{msg}}$, because an IR is frequently broadcasted. Note that the ASYNC scheme shows the lowest $M_{\text {msg }}$, when the update interval is very large $\left(T_{\text {update }}=1000\right)$. This is due to the fact that an IR is less frequently broadcasted, and less number of messages is used because more number of queried data items are received from the MTs. The ACOD scheme shows the lowest $M_{\mathrm{msg}}$ for the entire update intervals.

\section{Concluding remarks and future work}

In this paper, we investigated the cache invalidation problem in an IMANET, where the issues are different from that in a cellular network due to multi-hop message relay, operation cost model, and uncertainly in message delivery. In light on this, several push and pull-based cache invalidation strategies are carefully compared and analyzed. First, we proposed a GPS-based connectivity estimation (GPSCE) scheme as a base algorithm to support any cache invalidation strategy in IMANETS. Then, we proposed a pullbased cache invalidation policy, called ACOD. In addition, we extended two prior push-based cache invalidation strategies to work in IMANETS.

We conducted a simulation based performance study to examine the performance of different cache invalidation strategies. In the MTS and MTS + UIR schemes, there is an unavoidable delay before processing a query, since a request has to wait for the next IR/UIR. Also the message overhead due to broadcasted IR/UIRs leads to a waste of wireless bandwidth. The ACOD scheme provides high throughput (successful query), lowest query delay, and minimal communication overhead for different workload configurating. Thus, it is a viable approach for implementation in IMANETS.

There are many challenges that need further investigation to exploit the potential improvements in our study. Currently, we are examining the following issues:

- We plan to extend our scheme to handle an inaccurate location information measured by GPS device. In this paper, we assumed that the location information is accurate enough, but this assumption may not hold in a certain outdoor environment. Thus, we could relax this assumption. Also, it is interesting to incorporate with techniques in $[6,29]$.

- Since an energy conservation is one of the most critical issues in IMANETS, we plan to consider an energy efficient cache invalidation scheme. For example, due to multihop IRs and message relays, it needs to schedule the sleep and wake up modes of the MTs judiciously during the IR interval without degrading the performance.

\section{Acknowledgements}

This research was supported in part by NSF Grants CCR-0208734, CCF-0429631, EIA-0202007, and CNS 0509251. Wang-Chien Lee is supported in part by NSF Grants IIS-0328881, IIS-0534343, CNS-0626709. Preliminary results of this paper have been presented in part at IEEE MASS 2004 [22].

\section{References}

[1] NAVSTAR GPS Operations. http://tycho.usno.navy.mil/gpsinfo.html.

[2] A. Agarwal, S.R. Das, Dead Reckoning in Mobile Ad Hoc Networks, in: Proceedings of IEEE Wireless Communications and Networking Conference (WCNC 2003), 2003.

[3] D. Barbara, T. Imielinksi, Sleepers and Workaholics: Caching Strategies for Mobile Environments, in: Proceedings of ACM SIGMOD, 1994, pp. 1-12.

[4] M. Buddhikot, G. Chandranmenon, S. Han, Y.W. Lee, S. Miller, L. Salgarelli, Integration of 802.11 and Third-Generation Wireless Data Networks, in: Proceedings of IEEE INFOCOM, 2003.

[5] G. Cao, A scalable low-latency cache invalidation strategy for mobile environments, IEEE Transactions on Knowledge and Data Engineering 15 (5) (2003) 1251-1265.

[6] S. Capkun, M. Hamdi, J. Hubaux, GPS-free positioning in mobile adhoc networks, Cluster Computing Journal 5 (2) (2002) 118-124.

[7] K. Chen, K. Nahrstedt, Effective Location-Guided Tree Construction Algorithms for Small Group Multicast in MANET, in: Proceedings of IEEE INFOCOM, 2002, pp. 1180-1189.

[8] M.S. Corson, J.P. Macker, G.H. Cirincione, Internet-Based Mobile Ad Hoc Networking, in: IEEE Internet Computing, July-August 1999, pp. 63-70.

[9] R. Estrada, D. Munoz-Rodriquez, C. Molina, K. Basu, Cellular Position Location Techniques, a Parameter Detection Approach, in: Proceedings of IEEE International Vehicular technology Conference, 1999, pp. 1166-1171. 
[10] T. Hara, Effective Replica Allocation in Ad Hoc Networks for Improving Data Accessibility, in: Proceedings of IEEE INFOCOM, 2001, pp. 1568-1576.

[11] T. Hara, Replica Allocation in Ad Hoc Networks with Period Data Update, in: Proceedings of 3rd International Conference on Mobile Data Management (MDM), 2002, pp. 79-86.

[12] Q. Hu, D. Lee, Cache algorithms based on adaptive invalidation reports for mobile environments, Cluster Computing (1998) 39-48.

[13] Q. Hu, D.L. Lee, W. Lee, Performance Evaluation of a Wireless Hierarchical Data Dissemination System, in: Proceedings of ACM MOBICOM, 1999, pp. 163-173.

[14] S. Jiang, D. He, J. Rao, A Prediction-based Link Availability Estimation for Mobile Ad Hoc Networks, in: Proceedings of IEEE INFOCOM, 2001, pp. 1745-1752.

[15] J. Jing, A. Elmagarmid, A. Helal, R. Alons, Bit-sequences: an adaptive cache invalidation method in mobile client/server environment, Mobile Networks and Applications (1997) 115-127.

[16] D.B. Johnson, D.A. Maltz, Dynamic source routing in ad hoc wireless networks, in: Mobile Computing, Kluwer, 1996, pp. 153-181.

[17] A. Kahol, S. Khurana, S. Gupta, P. Srimani, An Efficient Cache Management Scheme for Mobile Environment, in: Proceedings of 20th International Conference on Distributied Computing System (ICDCS), April 2000, pp. 530-537.

[18] G. Karumanchi, S. Muralidharan, Ravi Prakash, Information Dissemination in Partitionable Mobile Ad Hoc Networks, in: Proceedings of IEEE Symposium on Reliable Distributed Systems (SRDS), 1999, pp. 4-13.

[19] M. Kazar, Synchronization and Caching Issues in the Andrew File System, in: Proceedings of USENIX Conference, 1988, pp. 27-36.

[20] S. Lee, W. Su, M. Gerla, Ad Hoc Wireless Multicast with Mobility Prediction, in: Proceedings of 8th International Conference on Computer Communications and Networks (ICCCN), October 1999, pp. 4-9.

[21] Q. Li, D. Rus, Sending Messages to Mobile Users in Disconnected Ad-hoc Wireless Networks, in: Proceedings of ACM MOBICOM, 2000, pp. 44-55.

[22] S. Lim, W. Lee, G. Cao, C.R. Das, Performance Comparison of Cache Invalidation Strategies for Internet-based Mobile Ad Hoc Networks, in: Proceedings of IEEE International Conference on Mobile Ad-hoc and Sensor Systems (MASS), 2004, pp. 104-113.

[23] S. Lim, W. Lee, G. Cao, C.R. Das, A novel caching scheme for improving internet-based mobile ad hoc networks performance, Ad Hoc Networks Journal 4 (2) (2006) 225-239.

[24] H. Luo, R. Ramjee, P. Sinha, L. Li, S. Lu. UCAN: A Unified Cellular and Ad-Hoc Network Architecture, in: Proceedings of ACM MOBICOM, 2003, pp. 353-367.

[25] J. Luo, J. Hubaux, P. Th. Eugster, PAN: Providing Reliable Storage in Mobile Ad Hoc Networks with Probabilistic Quorum Systems, in: Proceedings of ACM MobiHoc, 2003, pp. 1-12.

[26] M. Papadopouli, H. Schulzrinne, Effects of Power Conservation, Wireless Converage and Cooperation on Data Dissemination among Mobile Devices, in: Proceedings of MobiHoc, 2001, pp. 117-127.

[27] B.W. Parkingson, J.J. Spilker, Global Positioning System: Theory and Applications, 1 and 2, American Institute of Aeronautics and Astronautics, 1996

[28] T.S. Rappaport, Wireless communications: principles and practice, Prentice Hall, 1996.

[29] J.H. Reed, T.S. Rappaport, B.D. Woerner, Global positioning using wireless communications on highways of the future, IEEE Communications Magazine 34 (10) (1996) 33-41.

[30] M. Roussopoulos, M. Baker, CUP: Controlled Update Propagation in Peer-to-Peer Networks, in: Proceedings of USENIX Annual Technical Conference, 2003.

[31] F. Sailhan, V. Issarny, Cooperative Caching in Ad Hoc Networks, in: Proceedings of 4 th International Conference on Mobile Data Management (MDM), 2003, pp. 13-28.

[32] S. Sandberg, S. Kleiman D. Goldberg, D. Walsh, B. Lyon, Design and Implementation of the Sun Network File System, in: Proceedings of USENIX Summer Conference, 1985, pp. 119-130
[33] A.R. Shahani, D.K. Schaeffer, T.H. Lee, A $12 \mathrm{~mW}$ Wide Dynamic Range CMOS Front-end for a Portable GPS Receiver, in: Proceedings of IEEE International Solid-State Circuits Conference, 1997, pp. 368-369.

[34] Bruce Tuch, Development of WaveLAN an ISM band wireless LAN, AT\& T Techical Journal 72 (4) (1993) 27-33.

[35] K. Wu, P. Yu, M. Chen, Energy-Efficient Caching for Wireless Mobile Computing, in: Proceedings of 20th International Conference on Data Engineering, 1996, pp. 336-345.

[36] L. Xiao, L. Ni, A. Esfahania, Prioritized overlay multicast in mobile ad hoc environments, IEEE Computer 37 (2) (2004) 67-74.

[37] T. Ye, H.A. Jacobsen, R. Katz, Mobile Awareness in a Wide Area Wireless Network of Info-station, in: Proceedings of ACM MOBICOM, 1998, pp. 109-120.

[38] M.K.H. Yeung, Y.K. Kwok, Wireless cache invalidation schemes with link adaptation and downlink traffic, IEEE Transactions on Mobile Computing 4 (1) (2006) 68-83.

[39] L. Yin, G. Cao, Supporting cooperative caching in ad hoc networks, IEEE Transactions on Mobile Computing 5 (1) (2006) 77-89.

[40] W. Zhang, G. Cao, Defend Against Cache Consistency Attacks in Wireless Ad Hoc Networks, in: Proceedings of Mobile and Ubiquitous Systems (Mobiquitous), 2005, pp. 12-21.

[41] G.K. Zipf, Human behavior and the principle of least effort, AddisonWesley, Cambridge, MA, 1949.

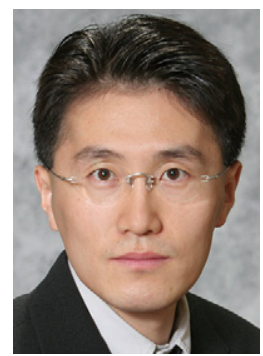

Sunho Lim received his B.S. degree (summa cum laude) in the Dept. of Computer Science and M.S. degree in the Dept. of Computer Engineering from Hankuk Aviation University, Korea, in 1996 and 1998, respectively. He received $\mathrm{Ph} . \mathrm{D}$. degree in the Dept. of Computer Science and Engineering from The Pennsylvania State University, University Park, in 2005. Since then, he has been an assistant professor in Software Engineering Program in the Dept. of Electrical Engineering and Computer Science at South Dakota State University. His research interests are in the areas of wireless networks and mobile computing, mobile data management, network security, and networked embedded systems. He was a recipient of the best graduate student teaching assistant award of the Dept. of Computer Science and Engineering at the Pennsylvania State University in 2002. He is a member of the IEEE.

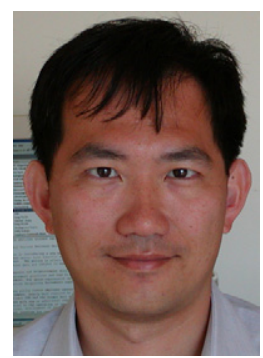

Wang-Chien Lee is an Associate Professor of Computer Science and Engineering at Pennsylvania State University. He received his B.S. from the Information Science Department, National Chiao Tung University, Taiwan, his M.S. from the Computer Science Department, Indiana University, and his Ph.D. from the Computer and Information Science Department, the Ohio State University. Prior to joining Penn State, he was a principal member of the technical staff at Verizon/GTE Laboratories, Inc. Dr. Lee leads the Pervasive Data Access (PDA) Research Group at Penn State University to perform cross-area research in database systems, pervasive/ mobile computing, and networking. He is particularly interested in developing data management techniques (including accessing, indexing, caching, aggregation, dissemination, and query processing) for supporting complex queries in a wide spectrum of networking and mobile environments such as peer-to-peer networks, mobile ad-hoc networks, wireless sensor networks, and wireless broadcast systems. Meanwhile, he has worked on XML, security, information integration/retrieval, and objectoriented databases. His research has been supported by NSF and industry grants. Most of his research result has been published in prestigious journals and conferences in the fields of databases, mobile computing and 
networking. He has served as a guest editor for several journal special issues on mobile database-related topics, including IEEE Transaction on Computer, IEEE Personal Communications Magazine, ACM MONET, and ACM WINET. He was the founding program committee co-chair for the International Conference on Mobile Data Management. He is a member of the IEEE and the Association for Computer Machinery.

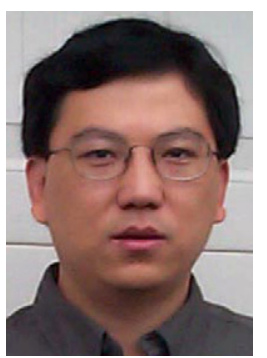

Guohong Cao received his BS degree from Xian Jiaotong University, Xian, China. He received the MS degree and Ph.D. degree in computer science from the Ohio State University in 1997 and 1999 respectively. Since then, he has been with the Department of Computer Science and Engineering at the Pennsylvania State University, where he is currently an Associate Professor. His research interests are wireless networks and mobile computing. He has published over one hundred papers in the areas of sensor networks, wireless network security, data dissemination, resource management, and distributed fault-tolerant computing. He is an editor of the IEEE Trans- actions on Mobile Computing and IEEE Transactions on Wireless Communications, and has served on the program committee of many conferences. He was a recipient of the NSF CAREER award in 2001.

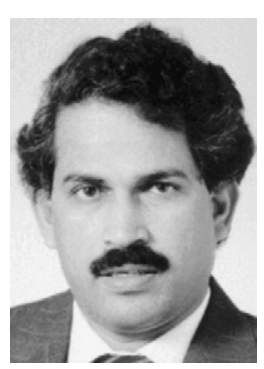

Chita R. Das received the M.Sc. degree in electrical engineering from the Regional Engineering College, Rourkela, India, in 1981, and the Ph.D. degree in computer science from the Center for Advanced Computer Studies, University of Louisiana, Lafayette, in 1986. Since 1986, he has been with the Pennsylvania State University, where he is currently a Professor in the Department of Computer Science and Engineering. His main areas of interest are parallel and distributed computer architectures, cluster computing, mobile computing, Internet QoS, multimedia systems, performance evaluation, and fault-tolerant computing. He has served on the Editorial Boards of the IEEE Transactions on Computers and IEEE Transactions on Parallel and Distributed Systems. Dr. Das is a Fellow of the IEEE and a member of the ACM. 